\title{
Mejorar la calidad de las evaluaciones de riesgos psicosociales mediante el control de sesgos
}

\author{
Improving the quality of psychosocial risk \\ assessments based on the identification and control \\ of bias
}

Rita Louzán Mariño ${ }^{1}$

Escuela Superior de Ingeniería y Tecnología, Universidad Internacional de La Rioja, Logroño, España

Fechas · Dates

Recibido: 2019.03.01

Aceptado: 2019.11.21

Publicado: 2020.01.15
Correspondencia · Corresponding Author

Rita Louzán Mariño

Escuela Superior de Ingeniería y Tecnología-ESIT. Universidad Internacional de La Rioja, Logroño (UNIR). Avenida de la Paz, 137, 26006, Logroño (La Rioja) Telf.: (+34)941210211Ｅmail: rita.louzan@unir.net 


\title{
Resumen
}

La evaluación de riesgos psicosociales de origen laboral constituye un fenómeno complejo y controvertido. Sus principales críticas están centradas en la validez de las herramientas que se emplean para la recogida de información, fundamentalmente autoinformes sensibles a diversas fuentes de error. Si bien se ha incrementado el esfuerzo por proporcionar evidencias sobre la fiabilidad y la validez de los instrumentos de evaluación disponibles, existen importantes fuentes de error, sesgos, que con frecuencia son ignoradas. La detección y control de sesgos es un tópico abordado desde varios ámbitos, entre ellos el de la psicología del trabajo, sin embargo, es desconocido por muchos técnicos de prevención de riesgos laborales. En este sentido, este artículo intenta acercar a los profesionales de la prevención de riesgos laborales, una mejor comprensión de este concepto, así como diversas técnicas para su identificación y control, en aras de mejorar la calidad de las evaluaciones de riesgo psicosocial.

Palabras clave: Riesgos laborales; estrés laboral; medición de riesgos; sesgo

\begin{abstract}
The assessment of workplace psychosocial risk factors is complex and controversial. The main criticism is focused on the validity of the tools used in their evaluation; basically, self-report measures which are subject to several sources of error. While there has been an increase in efforts to provide evidence on the reliability and the validity of the available assessment instruments, bias is often ignored as an important source of error. Although bias may be a novel topic to many prevention technicians, their detection and control is a traditional and well-studied theme in the field of work psychology. This article gives lay practitioners a better understanding of the concept of bias, as well as the related modes of identification and control, to improve the quality of psychosocial risk assessments.
\end{abstract}

Key words: occupational risk; occupational stress; risk assessment; bias 


\section{Introduccion}

La exposición del trabajador a factores psicosociales de riesgo, también llamados estresores, está relacionada con trastornos de la salud de muy diversa índole, tales como enfermedades cardiovasculares, trastornos músculoesqueléticos, alteraciones del sueño, depresión y suicidio, entre otros ${ }^{(1)}$. El peligro potencial de los estresores laborales obliga al empresario a incluirlos dentro de su plan de prevención de riesgos con objeto de eliminarlos o minimizarlos, tal y como recoge la Ley de Prevención de Riesgos Laborales (LPRL)(2). En este sentido, cobra vital importancia la evaluación de riesgos psicosociales, pues es se trata de la principal fuente de identificación y prevención de estos riesgos.

La ausencia de normativa específica sobre cómo evaluar los riesgos psicosociales ha favorecido la proliferación de herramientas de evaluación basadas en cuestionarios de autoinforme como principal instrumento de medida. Son de bajo coste, fáciles de aplicar e interpretar, y además están construidos con garantías psicométricas (i.e., fundamentación teórica, fiabilidad, validez y baremación), imprescindibles para proporcionar confianza en los resultados ${ }^{(4)}$. Con todo, para tratar de obtener mediciones que reflejen fielmente la realidad de la organización, es preciso tener en cuenta que el procedimiento de evaluación ha de carecer de sesgo ${ }^{(5,6)}$, entendiendo por sesgos «aquellos errores que modifican la información, alejándola de la realidad, y que son inherentes al instrumento usado en la observación o a la metodología usada en su aplicación»(7).

Cuestiones tales como la población a la que se dirige la prueba, las circunstancias de evaluación, el entrenamiento previo de la persona encargada de administrar los test o ítems demasiado largos son algunas de las muchas circunstancias generadoras de sesgos que pueden influir en la realidad de los resultados, afectando así a su validez ${ }^{(7,8)}$. Un ejemplo ilustrativo puede consultarse en la revisión realizada por Choi et al. ${ }^{(7)}$, que a modo de catálogo recoge 48 fuentes de error propias de los cuestionarios de salud, la gran mayoría fácilmente extrapolables a las herramientas que evalúan riesgos psicosociales. No podemos tampoco olvidar una de las advertencias más reiteradas por los expertos como es la posibilidad de falseamiento y distorsión de las respuestas por parte de los encuestados ${ }^{(9)}$. Por dichos motivos, la propia Fundación Europea ${ }^{(10)}$ desaconseja el uso de autoinformes en las evaluaciones de violencia y acoso laboral o sexual.

En relación a la investigación organizacional, uno de los sesgos más presentes se refiere a la deseabilidad social (DS), que puede definirse como la tendencia a responder a las preguntas del cuestionario en base a lo que es socialmente aceptado(11). En ese caso, la distorsión de las respuestas podría ser positiva o negativa, según lo que implique el contenido del ítem ${ }^{(12)}$. Un claro ejemplo es el relatado por Duro(12), quien indica que si contestamos de modo socialmente deseable a preguntas que lleven implícitas consecuencias negativas para la organización, como absentismo o impuntualidad, la mayoría de respuestas serían «nunca» o "casi nunca». En este caso, la mayor parte de contestaciones se concentrarían en las puntuaciones más bajas de la escala. Por el contrario, si el contenido del ítem alude a rasgos o actitudes del trabajador que favorecen su propia imagen o a la or- 
ganización, las contestaciones se aglutinarán en el extremo opuesto de la escala (e.g., «siempre», «casi siempre»).

A pesar de que la DS afecta a la validez de los resultados, pocas veces se tiene en cuenta en el ámbito organizacional(13) y mucho menos en la esfera relativa a la prevención de riesgos laborales. En consecuencia, podemos encontramos con estudios cuyos resultados peculiares solo son entendibles bajo el influjo de la DS y, por lo tanto, cuestionables. Un ejemplo se encuentra en el trabajo de Louzán(14), tras realizar una exploración del impacto de los factores psicosociales en una muestra de trabajadores del sector de la construcción, utilizando el método CoPsoQ-ISTAS 21, se halló que el nivel de exposición más desfavorable para la salud se encontraba en el factor de doble presencia (>45\%). Teniendo en cuenta que este estresor es catalogado como «específico de las mujeres» ${ }^{(15)}$, resulta curioso encontrar un porcentaje de exposición tan elevado cuando el 97\% de los participantes de dicha investigación han sido hombres. Ejemplos como éste ponen de manifiesto la necesidad de controlar los sesgos para disminuir el error de los resultados y, por consiguiente, incrementar su validez.

Existen diversas técnicas capaces de identificar, cuantificar y extraer la distorsión provocada por sesgos específicos, como la referida DS del ejemplo anterior. La escasa utilización de ellas en las evaluaciones de riesgo psicosocial podría deberse a su desconocimiento por parte de los Técnicos Superiores de Prevención de Riesgos Laborales (TSPRL), pues a pesar de ser conocidas en el campo de la evaluación psicológica y de existir considerable literatura científica al respecto ${ }^{(16)}$, pasan desapercibidas para muchos profesionales dedicados a la prevención, tal vez por falta de formación especializada en disciplinas como la evaluación psicológica o la psicometría ${ }^{(17)}$. La titulación en TSPRL es la única que capacita legalmente para realizar evaluaciones de riesgo psicosocial, tal y como recogen los artículos 37.1 y 37.2 del Reglamento de Servicios de Prevención (RSP)(18).

Este trabajo pretende acercar a los profesionales dedicados a la prevención de riesgos psicosociales una descripción de los sesgos más comunes y diferentes técnicas para su identificación y control, pues una estrategia para evitar los sesgos es conocerlos.

\section{Fuentes de error en las evaluaciones de riesgo psicosocial}

Para abordar el concepto de sesgo es preciso comenzar haciendo referencia a las fuentes de error achacables a la evaluación de riesgos psicosocial (Figura 1). Éstas pueden tener su origen en el objeto o sujeto a medir, en el mismo instrumento de medida utilizado, y/o en la persona que realiza la medición(20). Se clasifican en dos tipos: error aleatorio y error sistemático o sesgo (Figura 2). 


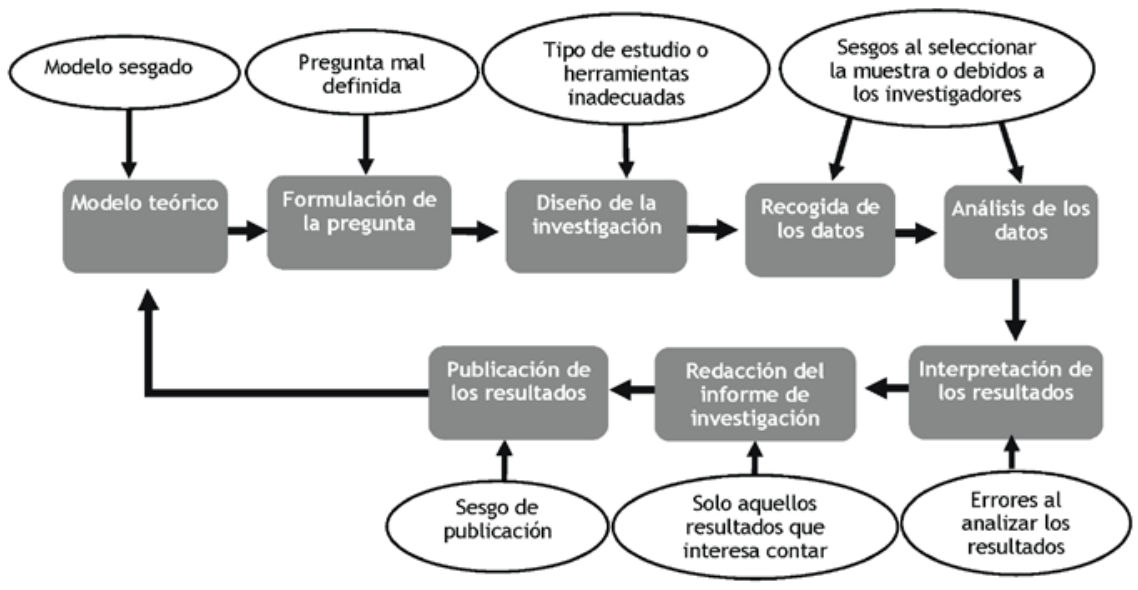

Figura 1: Errores y sesgos en las distintas fases de evaluación de riesgos psicosociales. Fuente: Salamanca AB. El "AEIOU" de la investigación en Enfermería. Madrid: FUDEN; 2013.

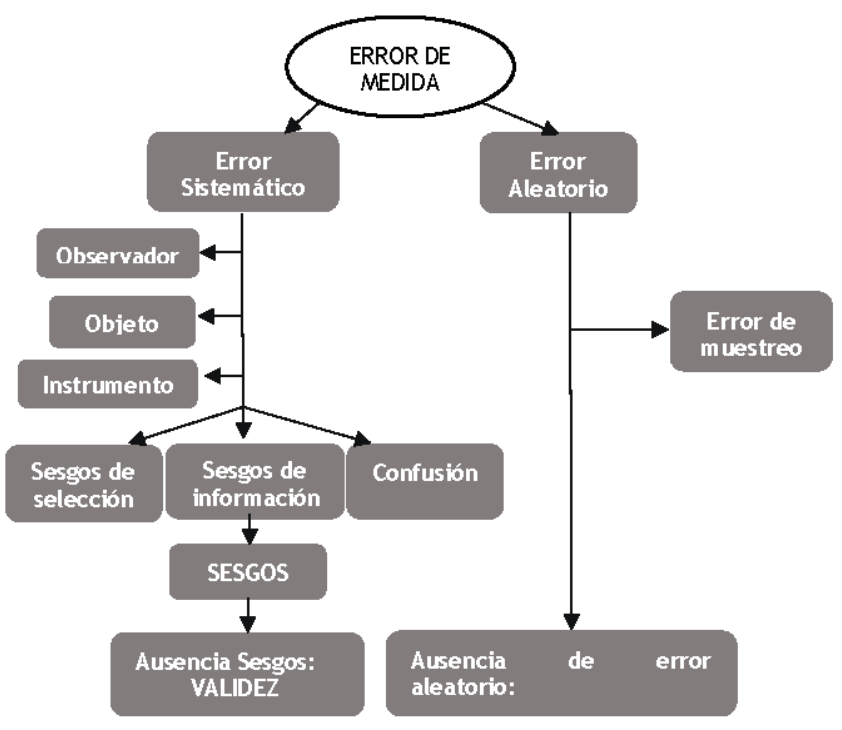

Figura 2: Errores de medida en los procesos de evaluación de riesgos psicosociales.

El tipo de error que interesa a este estudio es el sistemático, también denominado sesgo. El sesgo no es un error casual y estaría causado por algún factor que se ha obviado, como fallos en el diseño, en la conducción o en el análisis de una evaluación ${ }^{(5)}$. Los sesgos ocasionan desviaciones constantes en los resultados cada vez que se realiza la evaluación, sobre los mismos o distintos participantes, presentando valores más altos o más bajos que los reales ${ }^{(21)}$. Por ende, afecta a la validez de los resultados. Al ser un error que no depende del azar, no se reduciría al incrementar el tamaño de la muestra, sin embargo, conocida la causa del sesgo puede evitarse, corregirse o compensarse en buena parte. 


\section{Sesgos de respuesta en los tests de autoinforme}

De toda la tipología de sesgos que pueden afectar a los cuestionarios de autoinforme, los sesgos de respuesta son los de mayor impacto. Se refieren a la tendencia a responder a los ítems de un test sin tener en cuenta su contenido ${ }^{(22)}$. Cuando se responde a un ítem es preferible pensar que la respuesta depende exclusivamente de lo que se pregunta. Sin embargo, no siempre es así, ya que entran en juego otros factores como la propia formulación del ítem o la personalidad de quien responde ${ }^{(23)}$. Por ejemplo, «un individuo puede estar más inclinado a responder «sí» 0 «verdadero» en lugar de «no» 0 «falso»(24).

Los estilos de respuesta más habituales en la evaluación mediante autoinformes son la aquiescencia, la deseabilidad social, la tendencia central de respuesta y la severidad o tendencia extrema de respuesta (véase Man ${ }^{(25)}$, para una revisión más exhaustiva de los diferentes estilos de respuesta). De todos ellos, los más influyentes, y a la vez más estudiados, son la aquiescencia y la DS ${ }^{(26)}$, pudiendo actuar, incluso, de forma simultánea.

\section{Deseabilidad Social}

La DS es el sesgo que más literatura científica ha generado(27) y es considerado como una de las mayores amenazas en los estudios sobre psicología y ciencias sociales ${ }^{(13)}$. Algunos autores definen la DS como la tendencia de responder a un cuestionario de forma más favorable o menos favorable, según se crea que la respuesta pueda ser culturalmente mejor aceptadata ${ }^{(11)}$. Por ejemplo, ante la pregunta «Alguna vez he dicho algo malo de otra persona», responder negando este ítem aportaría una imagen positiva del encuestado ante los demás. De este modo, dependiendo de la impresión que se quiera ofrecer, la distorsión puede ser positiva o negativa.

El efecto de la DS ha sido sobradamente probado en cuestionarios de personalidad empleados en la práctica clínica, forense y médico-legal(28). Igualmente, se ha dejado constancia de su influencia en el campo de la psicología del trabajo y de las organizaciones ${ }^{(29)}$. Sirva de referencia el interesante estudio sobre estrés laboral de Semmer, Grebner y Elfering ${ }^{(30)}$, quienes corroboraron la susceptibilidad de los autoinformes a posibles asociaciones espurias (engañosas) entre las condiciones del trabajo y el bienestar laboral. En concreto, hallaron que las relaciones descritas estaban sesgadas debido a la DS y la variable de afectividad negativa. En la misma línea se encuentra el trabajo de Gamero-Burón y González ${ }^{(31)}$ al interpretar que los elevados índices de estrés laboral, recogidos en diversas fuentes (e.g., encuesta de calidad de vida en el trabajo, encuesta nacional de salud), se deben en buena parte al impacto de la DS. Según estos autores, experimentar estrés laboral sería la respuesta socialmente más aceptable, pues implicaría ser un trabajador productivo y, de ese modo, valioso para la organización. Ante estos datos, la reflexión es obvia: ¿los cuestionarios que evalúan riesgos psicosociales podrían ser sensibles al efecto de la DS? 


\section{Respuesta Aquiescente}

La aquiescencia (AQ) es entendida como la preferencia a contestar afirmativamente un cuestionario sin tener en cuenta el contenido de la pregunta, especialmente cuando se duda en la respuesta ${ }^{(32)}$. Couch y Keniston ${ }^{(33)}$, administrando cientos de ítems de una larga lista de escalas, mostraron dos tipos de tendencias de respuesta, los que siempre están de acuerdo con los ítems Yeasayers y los que siempre están en desacuerdo Naysayers. En la misma línea, otros autores realizaron la misma diferenciación ${ }^{(34,35)}$.

Varias investigaciones han demostrado que estimulan la respuesta aquiescente las siguientes circunstancias: (i) los ítems formulados en sentido positivo en comparación a los formulados en sentido inverso(23), (ii) el grado de ambigüedad de los ítems ${ }^{(23,36) ;}$ (iii) el formato de respuesta dicotómico (si-no; verdadero-falso); (iv) el tono en el que están formulados los ítems, y la redacción de los ítems de forma $\operatorname{vaga}^{(36)} ;(\mathrm{v})$ los ítems demasiado largos y complejos ${ }^{(23)}$ y $(\mathrm{vi})$ los temas sensibles ${ }^{(37)}$, entre otras.

\section{Tecnicas de control de deseabilidad y aquiescencia}

La presencia de sesgos en un test no implica necesariamente que toda la escala en su conjunto esté sesgada. Existen diferentes procedimientos para detectarlos, eliminarlos o equilibrarlos ${ }^{(38)}$. Lo deseable sería implementar el control de sesgos a la hora de diseñar la herramienta de evaluación, actuando por ejemplo en el estilo y redacción de los propios ítems del cuestionario, o incluyendo reactivos de control, entre otros recursos. No obstante, si esta fase ha sido obviada, como ocurre con la gran mayoría de escalas utilizadas en la evaluación de riesgos psicosociales, pueden aplicarse técnicas de control alternativas, una vez obtenida la información. Algunas de las soluciones más tradicionales se detallan en los párrafos precedentes.

\section{Control de la deseabilidad social en medidas de autoinforme}

Uno recurso muy utilizado para detectar y medir la DS es la aplicación de escalas específicas de medida. Al coexistir una gran diversidad de escalas ${ }^{(23,29)}$, la literatura científica es profusa. Entre las escalas más destacables se encuentran Marlowe-Crowne Social Desirability Scale (MCSDS)(39), las escalas L y K del Inventario Multifásico de Personalidad de Minnesota $(\mathrm{MMPI})^{(40)}$ y la escala de sinceridad de Eysenck ${ }^{(41)}$.

Estas escalas se aplican al mismo tiempo que la escala de interés, como podría ser el test CoPSoQ-ISTAS 21, o FPSICO, o DECORE, entre otras medidas; para luego comprobar si existe una asociación significativa entre ambas (e.g., a través del cálculo de correlaciones). En dicho caso se supone que operaría la DS y una de las 
posibles soluciones es eliminar las respuestas de los encuestados que puntúan alto en DS ${ }^{(11,23)}$ para evitar la influencia del sesgo en la interpretación de los datos.

La administración de escalas no agota las vías para el control de DS. Existen otras recomendaciones, que aunque no son siempre infalibles pueden minimizar las respuestas socialmente deseables si se utilizan de forma combinada ${ }^{(29)}$, a saber:

- Garantizar y enfatizar la confidencialidad y el anonimato. Cuanto más fácil sea identificar a los encuestados, más propensos serán éstos a dar respuestas deseables ${ }^{(23,42)}$.

- Administrar las pruebas vía internet. Las investigaciones revelan que las respuestas contienen menos DS porque la separación física entre la persona que aplica el test y el encuestado favorece el anonimato(43).

- Solicitar directamente sinceridad en las respuestas ${ }^{(42)}$.

- Informar de las consecuencias negativas para los distorsionadores de las respuestas $^{(29,44,45)}$.

- Crear baremos ${ }^{(29)}$.

Todas las estrategias de control de DS, hasta aquí citadas, son de fácil implementación, pero ninguna de ellas permite extraer un factor puro de DS. Para ello se recomienda utilizar técnicas más avanzadas como, por ejemplo, la propuesta de Ferrando(46). Este procedimiento está basado en análisis factoriales y consigue extraer puntuaciones individuales libres de sesgo. Si bien es una medida más compleja, evita el riesgo de eliminar información verdadera, como puede ocurrir al utilizar las escalas específicas de DS.

\section{Control de aquiescencia en medidas de autoinforme}

Al igual que ocurre con el control del sesgo de DS, también existen escalas que permiten controlar la aquiescencia como estilo de respuesta(47). Aunque han sido muy utilizadas durante la década de los 60 , sus bajas propiedades psicométricas han provocado su desuso, por lo que no son recomendadas para investigar tendencias generales de aquiescencia ${ }^{(23,35)}$.

La mayor parte de procedimientos recomendados para el control de la AQ están referidos a la redacción y construcción de los ítems de la escala de evaluación. Tanto es así, que son varios los autores que afirman que si se redactasen correctamente los instrumentos de medida el impacto de la aquiescencia sería insignificante ${ }^{(23)}$. Esta cuestión escapa a las competencias de los TSPRL, sin embargo, aunque participar en el diseño de las herramientas de evaluación psicosocial no sea su cometido, sí pueden elegir, de entre todos los instrumentos disponibles, aquellos que contengan el formato de ítems más apropiado para reducir las respuestas aquiescentes. Por ejemplo, pueden dar preferencia a las escalas que contengan balanceo de ítems ${ }^{(48)}$, en contraposición a las usuales escalas que contienen ítems en una sola dirección. Una escala balanceada significa que el 
cuestionario debe de tener la mitad de preguntas en el sentido a lo que se quiere medir y la otra mitad en el sentido opuesto.

Utilizando como ejemplo los dos primeros ítems de la versión corta del CoPSoQ-ISTAS 21, nos encontramos que en el primer ítem: «¿Con qué frecuencia la distribución de tareas es irregular y provoca que se te acumule el trabajo?», contestar con la opción de siempre significa obtener una puntuación de 4 puntos; sin embargo, contestar la misma opción en el ítem 2: «¿Con qué frecuencia tienes tiempo suficiente para hacer tu trabajo», supone una puntuación de 0 , es decir, es un ítem invertido. Es recomendable utilizar escalas blanceadas ${ }^{(23)}$ porque se formulan y responden con mayor atención, estimulando en menor medida la respuesta aquiescente. Además, si se poseen conocimientos estadísticos básicos, cuentan con la ventaja de permitir comprobar la correlación entre los ítems invertidos, y de ese modo se convierten en una medida de aquiescencia.

Otras cuestiones útiles para minimizar la respuesta aquiescente sería evitar las escalas que contengan: (i) muchos ítems, ya que propician la respuesta aquiescente por fatiga(26); (ii) enunciados complicados o demasiado vagos ${ }^{(36)}$; e (iii) ítems demasiado largos y con formato de respuesta dicotómico(36).

En el caso de tener que trabajar con una herramienta preexistente, sin posibilidad de seleccionar otra alternativa, siempre se podrían emplear medidas de control más avanzadas, basadas en análisis factorial, como las propuesta por Ferrando(46). La gran ventaja de utilizar esta metodología es que consigue calcular el error debido a AQ y DS y posteriormente lo elimina de los resultados. Dicho de otro modo, se consiguen extraer puntuaciones limpias de sesgo.

\section{Conclusiones y recomendaciones}

Ninguna evaluación es inmune a las fuentes de error, y menos cuando la información se recaba mediante cuestionarios autoadministrados. Por lo tanto, los test de evaluación de riesgos psicosociales no son ninguna excepción. Diferentes investigaciones así lo corroboran ${ }^{(30,31)}$ al apuntar directamente a la DS como uno de los principales responsables de las relaciones engañosas (espurias) encontradas al medir variables relacionadas con las condiciones de trabajo, el bienestar o el estrés laboral. Estas investigaciones evidencian que existe una posibilidad real de que los resultados obtenidos al evaluar condiciones de trabajo con autoinformes puedan estar sesgadas y, por ello, que las intervenciones que se deriven de tales evaluaciones puedan estar condenadas al fracaso al basarse en información errónea.

Sin embargo, los sesgos se pueden controlar. El primer paso para evitar su indeseable impacto es ser conscientes de su existencia, y desde esta premisa fue concebido este artículo, el cual ha pretendido acercar a los TSPRL una aproximación introductoria sobre las principales fuentes de error que acarrea el uso de cuestionarios en las evaluaciones psicosociales, así como algunas estrategias para su control. 
A la luz de este trabajo, los TSPRL deberían tener en cuenta que las fuentes de error pueden aparecer en cualquier fase de la evaluación de riesgos psicosocial, como por ejemplo en la fase de diseño (véase la figura 1). Para minimizar los errores en dicha fase, sería necesario revisar aspectos como el tipo de herramientas a utilizar, la delimitación precisa del contexto, las circunstancias y la población que se va a evaluar( ${ }^{(8)}$. Empero, controlar los sesgos en la fase de aplicación del test deja de ser una tarea sencilla, pues hay que tener en cuenta la variabilidad humana. Por una parte, los encuestados pueden malinterpretar las instrucciones de la prueba o equivocarse al marcar las respuestas, pueden estar influenciados por las condiciones ambientales del lugar donde realizan la prueba ${ }^{(8)}$, e incluso mentir ${ }^{(49)}$. Por otra parte, los propios evaluadores podrían equivocarse al realizar los análisis y/o malinterpretar los resultados e incluso subestimar los resultados no deseados.

Tras lo expuesto, resulta evidente que para evaluar las condiciones de trabajo no basta con administrar un cuestionario o transcribir la información saliente del software que corresponda. Hay que tratar de obtener mediciones fiables y válidas y, por este motivo se debe minimizar la varianza no deseada (los errores que alejan los resultados de la realidad), pues cuanto mayor sea el error más contaminado estarían los resultados del test. De este modo, identificar y controlar posibles sesgos estaría contribuyendo a mejorar la mencionada fiabilidad y la validez del instrumento utilizado(16). No obstante, esta cuestión colisiona con un inconveniente, ya señalado hace años por Meliá(17), que es la falta de formación especializada de gran parte de los TSPRL en disciplinas como la Teoría de los test o la evaluación psicológica, entre otras. De modo que, para llevar a cabo determinadas estrategias de control de sesgos como, por ejemplo, el método factorial propuesto por Ferrando ${ }^{(46)}$, que es capaz de limpiar la DS y la AQ de los resultados, será necesario poseer conocimientos avanzados de psicometría y técnicas estadísticas; disciplinas que, aunque tradicionales en evaluación psicológica, pueden suponer un tema novedoso para la mayoría de los TSPRL.

Con todo, para solventar la mayor parte de los problemas originados por la utilización de los cuestionarios de autoinforme existen una serie de recomendaciones internacionales que los TSPRL podrían seguir. Éstas son los estándares de buenas prácticas de AERA, et al. ${ }^{(8)}$ y de la Comisión Internacional de Test (ITC) ${ }^{(50)}$. Sus directrices ya operan en disciplinas como la psicología o la educación, ofreciendo pautas que van desde cómo construir y adaptar las pruebas hasta orientaciones sobre su administración y corrección. Entre algunas de sus recomendaciones se encuentran entrenar previamente al evaluador, preparar con antelación el lugar de la prueba cerciorándose de que sea cómodo y esté libre de distracciones, procurar reducir los niveles de ansiedad de los encuestados, eliminar los posibles focos de desviación de la atención como alarmas o teléfonos móviles o leer las instrucciones pausada y claramente. El objetivo es guiar a los evaluadores hacia estudios libres de sesgos, por este motivo conviene seguir estas directrices en los procesos de evaluación de riesgos psicosociales que utilicen el autoinforme como herramienta de medida. 
Una vez conocida la influencia que pueden llegar a tener los sesgos en los resultados de cualquier evaluación y sabiendo que existen diversidad de técnicas para evitarlos o reducirlos, lo adecuado, ética y profesionalmente, es tenerlos en cuenta. Por último, conviene precisar que los TSPRL son los únicos que legalmente pueden realizar evaluaciones de las condiciones de trabajo de carácter psicosocial. Precisamente, apelando a la responsabilidad que conlleva esta exclusividad en la recogida de información, se anima al profesional dedicado a la prevención de los riesgos psicosociales a profundizar en esta materia y llevarla a la práctica. En conclusión, lo que se pretende es conseguir la mayor calidad posible de los instrumentos de recolección de datos, pues ello redundará en la eficiencia de la prevención de riesgos y por consiguiente en la salud del trabajador y la productividad de las organizaciones.

\section{Referencias}

1. O.I.T. Estrés en el trabajo: un reto colectivo. Turín: Centro internacional de formación de la OIT; 2016.

2. Ley de Prevención de Riesgos Laborales. L. № 31/1995. (8 Nov 19953. Ferrer R. La evaluación de riesgos psicosociales en tiempos de crisis. Rev Prevención Riesgos Psicosoc y bienestar en el Trab. 2010;(2):67-85.

3. Ferrer R. La evaluación de riesgos psicosociales en tiempos de crisis. Rev Prevención Riesgos Psicosoc y bienestar en el Trab. 2010: 67-85.

4. Inspección del Trabajo y de la Seguridad Social (ITSS). Guía de actuaciones de la inspección de trabajo y seguridad social sobre riesgos psicosociales [internet]. Madrid: Ministerio de Empleo y Seguridad Social; 2012 [citado 2019 mar 19]. Disponible en: http://www.mitramiss.gob.es/itss/web/Atencion_al_Ciudadano/Normativa/DOCUM_ITSS/index.html

5. Gómez-Benito J, Hidalgo MD, Guilera G. El sesgo de los instrumentos de medición. Tests justos. Papeles del Psicólogo [internet]. 2010 [citado 2019 marz 19]; 31(1):75-84. Disponible en: http://papelesdelpsicologo.es/pdf/1798.pdf.

6. Ponsoda V. Metodología al servicio del psicólogo. Papeles del Psicólogo [internet]. 2009 [citado 2019 mar 19]; 31:2-6. Disponible en: http://www.papelesdelpsicologo.es/pdf/1137.pdf.

7. Choi B, Granero R, Pak A. Catálogo de sesgos o errores en cuestionarios sobre salud. Rev Costarric Salud Pública. 2010; 2: 106-118.

8. American Educational Research Association (AERA), American Psychological Association (APA), National Council on Measurement in Education (NCME). Standards for educational and psychological testing. $3^{\text {a }}$. ed. Washington, DC: American Educational Research Association.; 2014. [citado en 21 mar 2019]. Disponible en: https://eric.ed.gov/?id=ED565876.

9. Eysenck MW. Psicología básica. 1ª. Ciudad de México: Manual Moderno; 2019. 
10. Giaccone M, Nunzio D, Fromm A, Vargas O. Violence and harassment in European workplaces: causes, impacts and policies. Dublin; 2015. doi:10.5281/zenodo. 16387

11. Tracey TJG. A note on socially desirable responding. J Couns Psychol. 2016; 63: 224-232. doi:10.1037/cou0000135

12. Duro A. Calidad de vida laboral y psicología social de la salud laboral: hacia un modelo de componentes comunes para explicar el bienestar laboral psicológico y la salud mental laboral de origen psicosocial. Fundamentos teóricos. Rev del Minist Trab y Asuntos Soc Ser Econ y Sociol. 2005; 56: 15-56.

13. King MF, Bruner GC. Social desirability bias: A neglected aspect of validity testing. Psychol Mark. 2000; 17: 79-103. doi:10.1002/(SICI)1520-6793(200002)17:2< 79::AID-MAR2>3.0.C0;2-0

14. Louzán R. Exposición a los factores psicosociales adversos en una muestra de trabajadores de la construcción de la provincia de Lugo. Segur y salud en el Trab. 2014; 77: 28-35.

15. Rivas M. Salud y género: perspectiva de género en la salud laboral. Rev del Minist Trab e Inmigr. 2008; 74: 227-286.

16. Dupuis M, Meier E, Capel R, Gendre F. Measuring individuals' response quality in self-administered psychological tests: an introduction to Gendre's functional method. Front Psychol. 2015; 6: 1-12. doi:10.3389/fpsyg.2015.00629

17. Meliá JL. ¿Cómo evaluar los riesgos psicosociales en la empresa? Metodologías, oportunidades y tendencias. En: Mondelo, P, Mattila M, Karwowski W, Hale A, editores. Proceedings of the Fourth International Conference on ORP; 2006.

18. Reglamento de los Servicios de Prevención. R.D. № 39/1997 (17 Nov 1997).

19. Moreno B, Báez C. Factores y riesgos psicosociales, medidas y buenas prácticas [internet]. Madrid: INSHT; 2010 [citado 2019 mar 19]. 188 p. Disponible en: https://www.insst.es

20. de la Guardia G, Gómez ML, Sandoval E, García G. Sesgo o error de medición. En: Villa A, editor. Epidemiología y estadística en salud pública. México: McGraw. Hill; 2012:151-163.

21. Porta MS, editor. A dictionary of epidemiology (DRAFT). 6 ${ }^{\mathrm{a}}$. ed. New York: Oxford University Press; 2014. doi:10.1093/acref/9780199976720.001.0001

22. Peer E, Gamliel E. Too reliable to be true ? Response bias as a potential source of inflation in paper-and-pencil questionnaire reliability. A peer-reviewed Electron $\mathrm{J}$. 2011; 16: 1-8.

23. Morales P. Medición de actitudes en psicología y educación. Construcción de escalas y problemas metodológicos. $3^{\mathrm{a}}$ ed. Madrid: Universidad Pontificia de Comillas; 2006.

24. Cohen RJ, Swerdlik ME. Pruebas y evaluación psicológicas. México D.C.: McGraw-Hill; 2006. 
25. Man TS, Tsang SM. Do personality and self-construal predict response style in self- rating scales? Discov Student E-Jounal. 2013; 2: 29-48.

26. Ferrando PJ, Anguiano-Carrasco C. Response certainly, conscienciousness, and self-concept clarity as antecedents of acquiescence: A prediction model. Anu Psicol. 2012; 42: 103-112.

27. Cosentino AC, Castro A. Adaptación y validación argentina de la Marlowe-Crowne Social Desirability Scale. Interdisciplinaria. 2008; 25: 197-216

28. González H, Iruarrizaga I. evaluación de las distorsiones de respuesta mediante el MMPI-2. Papeles del Psicólogo. 2005; 26: 129-137.

29. Salgado JF. Personalidad y deseabilidad social en contextos organizacionales: implicaciones para la práctica de la psicología del trabajo y las organizaciones. Papeles del Psicólogo. 2005; 26:115-128.

30. Semmer NK, Grebner S, Elfering A. Beyond self-report: using and situation-based Measures physiological, observational, in research on occupational stress. En: Perrew P, Ganster D, editores. Emotional and physiological processes and positive intervention strategies. Research in occupational stress and well-being. Boston: Elsevier Ltd; 2004: 205-263. doi:10.1016/S1479-3555(03)03006-3

31. Gamero-Burón C, González ML, editores. Costes socio-económicos de los riesgos psicosociales. Balance de situación actual y propuestas metodológicas para avanzar en su medición o cálculo. Universidad de Málaga: Secretaría de Salud Laboral y Medio ambiente (UGT -CEC); 2013.

32. Billiet JB, Davidov E. Testing the stability of an acquiescence style factor behind two interrelated substantive variables in a panel design. Sociol Methods Res. 2008; 36: 542-562. doi:10.1177/0049124107313901

33. Couch A, Keniston K. Yeasayers and naysayers: Agreeing response set as a personality variable. J Abnorm Soc Psychol. 1960;60:151-174. doi:10.1037/h0040372

34. Baumgartner H, Steenkamp J-BEM. Response styles in marketing research: A cross-national investigation. J Mark Res. 2001; 38: 143-156. doi:10.1509/ jmkr.38.2.143.1884

35. Paulhus DL. Measurement and control of response bias. En: Robinson JP, Shaver PR, Wrightsman SL, editores. Cap. 2, Measures of personality and social psychological attitudes. San Diego, CA: Academic Press; 1991:17-59. doi: psycinfo/1991-97206-001.

36. Finlay WML, Lyons E. Acquiescence in Interviews With People Who Have Mental Retardation. Ment Retard. 2002;40:14-29. doi:10.1352/0047-6765(2002)040<0 014:AIIWPW>2.0.CO;2

37. Gove WR, Geerken MR. Response bias in surveys of mental health: An empirical investigation. Am J Sociol. 1977; 82: 1289-1317. 
38. Barker C, Pistrang N, Elliott R. Research methods in clinical psychology : an introduction for students and practitioners. $3^{\mathrm{a}}$ ed. Chichester, UK: Wiley-Blackwell; 2015.

39. Gutiérrez S, Sanz J, Espinosa R, Gesteira C, García-Vera MP. La escala de deseabilidad social de Marlowe-Crowne: baremos para la población general española y desarrollo de una versión breve. An Psicol. 2016; 32: 206-217. doi:10.6018/ analesps.32.1.185471

40. Hathaway SR, McKinley JC. The MMPI manual. New York: The Psychological Corporation; 1951

41. Eysenck HJ, Eysenck SBG. The Eysenck Personality Inventory manual. London: Routledge; 1964.

42. Podsakoff PM, MacKenzie SB, Lee J-Y, Podsakoff NP. Common method biases in behavioral research: a critical review of the literature and recommended remedies. J Appl Psychol. 2003; 88: 879-903. doi:10.1037/0021-9010.88.5.879

43. Risko EF, Quilty LC, Oakman JM. Socially desirable responding on the web: Investigating the candor hypothesis. J Pers Assess. 2006; 87: 269-276. doi:10.1207/ s15327752jpa8703_08

44. Converse PD, Oswald FL, Imus A, Hedricks C, Roy R, Butera H. Comparing personality test formats and warnings: Effects on criterion-related validity and test-taker reactions. Int J Sel Assess. 2008; 16: 155-169. doi:10.1111/j.14682389.2008.00420.x

45. Robson SM, Jones A, Abraham J. Personality, faking, and convergent validity: A warning concerning warning statements. Hum Perform. 2008 ;21: 89-106. doi:10.1080/08959280701522155

46. Ferrando PJ, Lorenzo-Seva U, Chico E. A general factor-analytic procedure for assessing response bias in questionnaire measures. Struct Equ Model A Multidiscip J. 2009; 16: 364-381. doi:10.1080/10705510902751374

47. Jackson DN, Messick S. Acquiescence and desirability as response determinants on the MMPI. Educ Psychol Meas. 1961; 21: 771-790. doi:10.1177/001316446102100402

48. Ferrando PJ, Demestre J, Anguiano-Carrasco C, Chico E. An IRT assessment of rotter I-E scale: A new approach and some considerations. Psicothema. 2011; 23: $282-288$.

49. Martínez-Selva J. La psicología de la mentira. Barcelona: Paidos; 2005.

50. Comisión Internacional de Tests (ITC). Directrices internacionales para el uso de los tests. INFOCOP [internet]. 2019 [citado 2019 mar 22]; 77:21-32. Disponible en: https://www.cop.es/index.php?page=directrices-internacionale.

51. Salamanca AB. El «AEIOU» de la investigación en Enfermería. Madrid: FUDEN; 2013. 